Cite this: RSC Advances, 2013, 3, 13115

Received 21st May 2013,

Accepted 10th June 2013

DOI: $10.1039 / \mathrm{c} 3 \mathrm{ra} 42505 \mathrm{c}$

\title{
High yield DNA fragmentation using cyclical hydrodynamic shearing
}

\author{
Lingling Shui, ${ }^{\text {ab }}$ Wouter Sparreboom, ${ }^{a}$ Peter Spang, ${ }^{\mathrm{c}}$ Tina Roeser, ${ }^{\mathrm{d}}$ Benjamin Nieto, ${ }^{\mathrm{e}}$ \\ Francesc Guasch, ${ }^{e}$ Antoni Homs Corbera, ${ }^{f}$ Albert van den Berg ${ }^{a}$ and Edwin \\ T. Carlen*a
}

systems, miniaturized lab-on-a-chip (LOC) platforms are attractive due to their ability to process small sample volumes and potential for multiplexed processing and high throughput analysis. Although many conventional sample preparation steps have been realized in LOC systems, only a few research groups have reported DNA fragmentation in a microfluidic chip..$^{2-5}$

Different DNA fragmentation techniques are available, such as enzymatic digestion, sonication, nebulization and hydrodynamic shearing. ${ }^{6}$ Both sonication and hydrodynamic shearing instruments are commercially available for the fragmentation of genomic DNA, ${ }^{7,8}$ however, are not suitable for small sample volumes and integration with other sample processing steps. Hydrodynamic shearing is reported to be one of the most robust DNA fragmentation techniques for the generation of size specific and small fragment lengths, with narrow size range, and limited end damage. ${ }^{8}$ Microfluidic LOC systems are particularly wellsuited for the hydrodynamic shearing of DNA since the fragmentation process is achieved by passing the DNA molecules through a constriction placed in a larger flow channel, which can be precisely manufactured using conventional microfabrication, molding and bonding techniques.

Fig. 1 shows a schematic drawing of a typical hydrodynamic shearing structure that contains a constriction channel embedded in a larger spacer channel. DNA molecules introduced from the left side of the channel (inlet pressure $P_{1}$ ) are transported with sample flow rate $Q$, due to pressure difference $\Delta P=P_{1}-P_{2}$, from the spacer channel to the constriction channel. The abrupt change in cross-sectional area from the spacer channel to the constriction

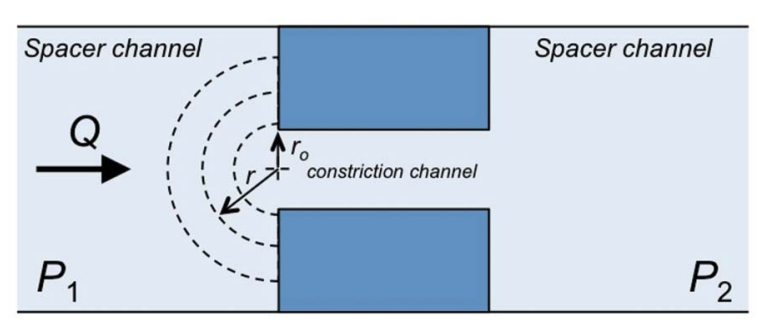

Fig. 1 Single constriction fragmentation structure.

\section{incrossectional area from the spacer channel to the constiction}

\footnotetext{
${ }^{a}$ BIOS/Lab on a Chip Group, MESA+ Institute for Nanotechnology, University of Twente, Postbus 217, 7500 AE Enschede, The Netherlands.

E-mail: e.t.carlen@utwente.nl; Fax: +31 (0)53 489 3595; Tel: +31 (0)53 4895399

${ }^{b}$ Institute of Electronic Paper Displays, South China Academy of Advanced Optoelectronics, South China Normal University, Guangzhou, China.

${ }^{c}$ Institut fur Mikrotechnik Mainz, Carl-Zeiss-Strasse 18-20, 55129 Mainz, Germany. E-mail: spang@imm-mainz.de; Fax:+49 6131/990-205; Tel: +49 6131/990-0

${ }^{d}$ thinXXS Microtechnology AG, Amerikastrasse 21, 66482 Zweibruecken, Germany. E-mail: roeser@thinxxs.de; Fax: +49 6332/8002-75; Tel: +49 6332/8002-33

${ }^{e}$ Biokit, S.A, 08186 Lilca d'Amunt, Barcelona, Spain. E-mail: fguasch@biokit.com; Fax: +34 (0)93 860 9017; Tel: +34 (0)93 8609000

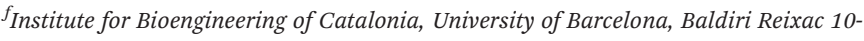
12, 08028 Barcelona, Spain. E-mail: ahoms@ibecbarcelona.eu; Fax: +34 (0)93 403 9702; Tel: +34 (0)934039706
} 
channel results in a large shear stress in the incompressible fluid near the constriction entrance, which creates extensional strain forces on the DNA molecules due to hydrodynamic drag forces. The DNA molecules undergo coil-stretch transitions in the extensional flow field and ultimately the molecular bonds break due to double-chain scission at a critical strain rate. ${ }^{9-15}$

Assuming that the sample flow can be modelled as a point-sink flow field, the fluid strain rate is $\mathrm{d} \varepsilon / \mathrm{d} t \equiv \mathrm{d} v / \mathrm{d} r \sim Q / r^{3}$, where $\mathrm{d} v / \mathrm{d} r$ is the flow velocity gradient, $Q$ is the volumetric flow rate, and $r$ is the radial distance from the spacer channel to the constriction channel. ${ }^{7,9}$ Since the constriction entrance has a finite radius $r_{\mathrm{o}}$, then the maximum strain rate is assumed to occur at $r=r_{\mathrm{o}}$. The final fragment length is ultimately determined by the flow rate of the fluid and the size and geometry of the transition from the spacer channel to the constriction channel.

We previously reported a microfluidic DNA fragmentation chip capable of producing short fragment lengths using a low-pressure hydrodynamic shearing configuration. ${ }^{2}$ It is well known that the fragmentation yield and size range can be improved through multiple sample recirculation cycles ${ }^{8,16}$ and the fragmentation yield was shown to improve from about $10 \%$ to over $60 \%$ after multiple manual recirculation cycles. ${ }^{2}$ Sample recirculation in short constrictions can reduce fragment length and size range, and improve yield, however, the recirculation process is time consuming, adds additional cost and complexity to automated fragmentation systems due to high pressure sample switching, and reduces the sample throughput. ${ }^{16}$

In this communication, we report a new high yield genomic DNA fragmentation technique based on cyclical hydrodynamic shearing. We consider only the fragment yield for a particular AFL using previously reported fragmentation concepts. ${ }^{2-4,7}$ The new fragmentation device uses a chain of series connected constriction channels with cross-sectional area $A_{\mathrm{c}}$, separated by the spacer channels with larger cross-sectional area $A_{\mathrm{s}}$. Note, the Reynolds number $R e \gg 1$ for the experimental conditions used in this article, and therefore, the Hagen-Poiseuille law for the estimation of hydraulic resistance is no longer valid, and therefore, the crosssectional area ratio $\gamma \equiv A_{\mathrm{s}} / A_{\mathrm{c}}$ has been used as the scaling parameter from our previous designs. ${ }^{2}$ For proper operation, adjacent constriction channels must be separated by sufficient length such that the sample fluid velocity decreases significantly before entering of the next adjacent constriction channel in the chain where the sample is accelerated due to the large $\gamma$. Repeated fluid velocity acceleration-deceleration cycles, as the sample moves through the microfluidic device, produces a similar effect as sample recirculation. This new approach is important, as it does not require external valves for sample switching and can be accomplished with a single injection cycle in the passive microfluidic device. It is important that the multi-constriction channel is designed such that the total hydraulic resistance is not prohibitively large, which limits $Q$.

Fig. 2a shows a schematic of the cyclical fragmentation microfluidic chip consisting of series connected constriction channels of length $L_{\mathrm{c}}$ that are each separated by a larger spacer channel of length $L_{\mathrm{s}}$. For these designs $\gamma \geqslant 10$, where $A_{\mathrm{s}}=w_{\mathrm{s}} h_{\mathrm{s}}$ and $A_{\mathrm{c}}=w_{\mathrm{c}} h_{\mathrm{c}}$, and $L_{\mathrm{c}}=0.5 \mathrm{~mm}$ from previous experiments. ${ }^{2}$ Note,

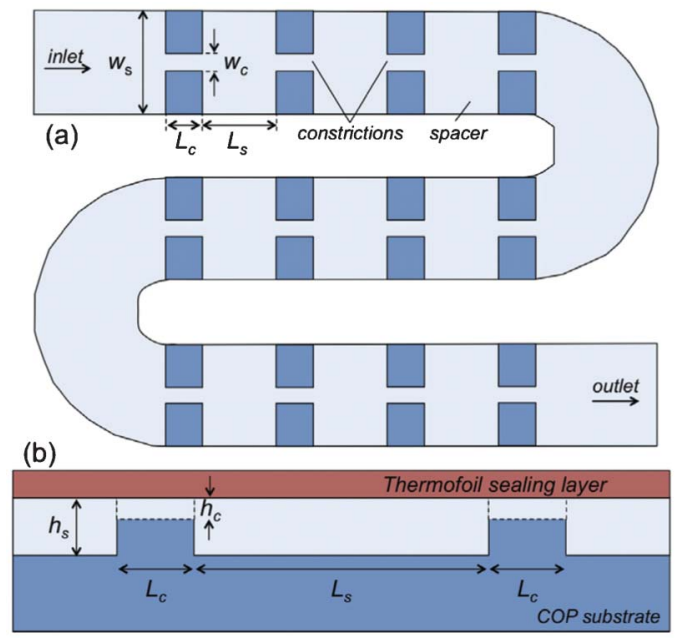

Fig. 2 (a) Top-view schematic of multiple constriction DNA fragmentation chip; (b) Side-view schematic of multi-height COP substrate with polymer foil to seal the channels.

that the constriction channel is typically shorter than the entrance length required for a fully developed parabolic flow velocity profile, ${ }^{17}$ thus it is expected that $L_{\mathrm{c}}$ can be decreased further, which will permit larger flow rates, i.e. smaller $\Delta P$, and improved fragmentation performance. The serpentine channel configuration was used to save areal space on the polymer substrates, and other configurations are possible.

The channel layer has been manufactured from cyclo-olefinpolymer (COP, Zeonor 1420R), which is commonly used for LOC applications since it is biocompatible, has low background fluorescence and is compatible with low cost high-throughput manufacturing, such as injection molding. A conventional DNA fragmentation chip manufactured with cyclo-olefin-coploymer (COC) has been previously reported. ${ }^{4}$ The multi-height channels were formed using conventional multi-height CNC milling (PICOMAX 60-HSC, Fehlmann AG), shown schematically in Fig. 2b. The channel layer is sealed with an optically clear pressure-sensitive adhesive polymer foil (Thermo Scientific Absolute ${ }^{\mathrm{TM}}$ QPCR Seal, thickness: $0.1 \mathrm{~mm}$ ) by placing the foil on top of the chip surface and applying a uniform pressure. Fig. 3 shows examples of manufactured chips. The minimum feature size achievable with this CNC instrument is about $0.1 \mathrm{~mm}$, however, plastic molding techniques are available that can produce smaller feature sizes, thus allowing for smaller sample volumes. A polymer sealing foil was chosen for this application due to its low cost and compatibility with high-throughput manufacturing, however, the bonded interface is not stable for large $Q$ and $\Delta P$ and limited the average fragment lengths (AFL) to $\sim 5 \mathrm{kbp}$, which is suitable for our diagnostic application.

Therefore, the testing of the devices was limited to flow rates that did not generate $\Delta P$ more than a couple bar, however, it is important to note that the thermofoil sealing layer used in this article can easily be replaced with well bonded thick polymer layers that can withstand much larger $Q$ and $\Delta P$, and sub-kbp fragment lengths. ${ }^{2,6}$ Fig. 3a shows an example of a manufactured 

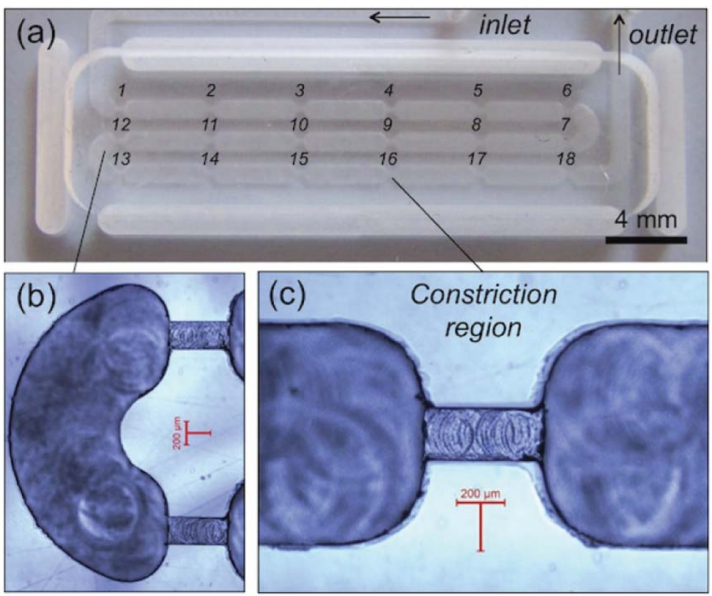

Fig. 3 Microscope images of manufactured fragmentation chips. (a) Top-view of fragmentation chip; (b) channel bend region; (c) constriction channel.

COP fragmentation chip prior to testing. Fig. $3 \mathrm{~b}$ and $3 \mathrm{c}$ show examples of the milled constriction regions in the COP substrate. The average surface roughness of the milled channels is $R_{\mathrm{a}} \approx 300$ $\mathrm{nm}$ (Fig. $3 \mathrm{~b}$ and $3 \mathrm{c}$ ), which is 1-2 orders of magnitude smaller the channel dimension and does not affect the performance of the device since the fragmentation process occurs near the center of the flow stream where $d v / d r$ is largest, which has been confirmed based on comparisons of fragmentation performance in glass channels with smooth surfaces. ${ }^{2}$

The spacing length $L_{\mathrm{S}}$ between adjacent constrictions (Fig. 2) required for the flow velocity to decrease sufficiently was determined by analysing the flow velocity $v_{\mathrm{x}}$ along the length of the channel in the $x$-direction using a three-dimensional finite element model (Multiphysics, Comsol), as shown in Fig. 4a. Fig. $4 \mathrm{~b}$ shows velocity $v_{\mathrm{x}}$ as a function of the channel length in the $x$-direction at $z=0.25 \mathrm{~mm}$. The flow accelerates from $v_{\mathrm{x}} \approx 0.2 \mathrm{~m}$ $\mathrm{s}^{-1}$ to $v_{\mathrm{x}} \approx 3.5 \mathrm{~m} \mathrm{~s}^{-1}\left(x_{1}\right)$ at the first constriction and subsequently decelerates to $v_{\mathrm{x}} \approx 0.6 \mathrm{~m} \mathrm{~s}^{-1}$ after a length $L_{\mathrm{s}} \approx 4 \mathrm{~mm}\left(x_{2}\right)$. The

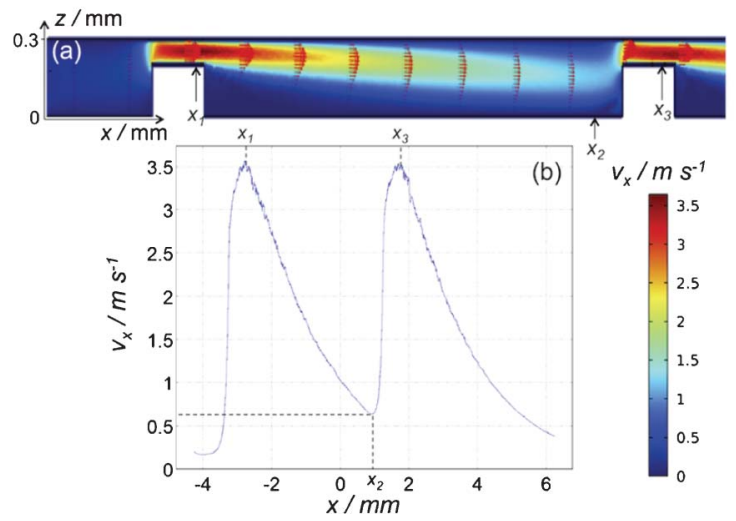

Fig. 4 Three-dimensional finite element simulation results. (a) Length cross-section showing fluid velocity magnitude as a function length in the $x$-direction and height in the $z$-direction along the length of the channel; (b) fluid velocity along the structure length at $z=0.25 \mathrm{~mm}$.
Table 1 Fragmentation device dimensions. All dimensions in $\mathrm{mm}$

\begin{tabular}{lcclllll}
\hline Design & $L_{\mathrm{c}}$ & $L_{\mathrm{s}}$ & $h_{\mathrm{c}}$ & $w_{\mathrm{c}}$ & $h_{\mathrm{s}}$ & $w_{\mathrm{s}}$ & $N$ \\
\hline $\mathrm{A}$ & 0.5 & 4.0 & 0.1 & 0.2 & 0.3 & 0.9 & 18 \\
$\mathrm{~B}$ & 0.5 & 4.0 & 0.2 & 0.3 & 0.5 & 1.3 & 18 \\
$\mathrm{C}$ & 0.5 & 4.0 & 0.15 & 0.3 & 0.5 & 1.3 & 18 \\
$\mathrm{D}$ & 0.5 & 8.5 & 0.2 & 0.3 & 0.5 & 1.3 & 9
\end{tabular}

flow accelerates again to $v_{\mathrm{x}} \approx 3.5 \mathrm{~m} \mathrm{~s}^{-1}\left(x_{3}\right)$ at the second constriction. The successive acceleration cycles provide a similar effect as sample recirculation. Table 1 lists the structure dimensions of four designs that have been fabricated and tested.

All experiments were conducted with salmon sperm DNA (D1626, deoxyribonucleic acid sodium salt from salmon testes, Sigma-Aldrich). The solid DNA sample was dissolved in deionized water $(>18 \mathrm{M} \Omega \mathrm{cm})$ in a plastic tube at a concentration of $1 \mathrm{mg}$ $\mathrm{ml}^{-1}$ and stored at $-20{ }^{\circ} \mathrm{C}$. Prior to experiments the DNA solution was thawed and diluted to the testing concentration. The nonfragmented (NF) DNA sample was tested with gel electrophoresis separations to confirm that no fragments less than $10 \mathrm{kbp}$ in length were present (Fig. 5a). The fragmentation chip was mounted into a custom-made chip-holder and connected to a gas-tight syringe (1725N, $1 \mathrm{ml}$, Hamilton) driven by a syringe pump (PHD2000, Harvard). Experiments were conducted with different sample flow rates $Q$ and designs with different dimensions. Prior to all experiments, the device was rinsed with ethanol and copious amounts of deionized water. The DNA was fragmented by pumping the sample into the microfluidic device at a flow rate such that $\Delta P$ did not exceed a few bar. After each fragmentation run, the microfluidic chip was washed with copious amounts of deionized water. The AFL were determined using gel electrophoresis separations. The fragmented DNA yields were estimated using densitometry (Labworks 4.0, UVP). Fig. 5 and

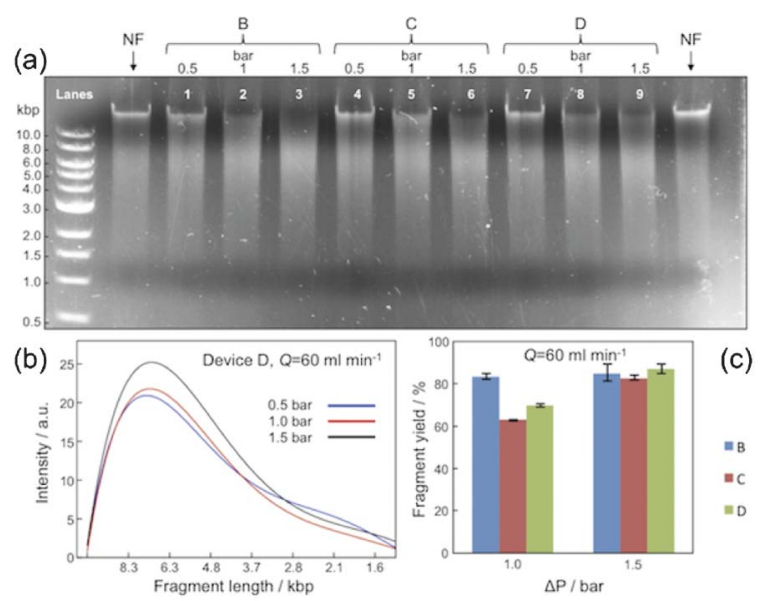

Fig. 5 Example measurement results for $Q=60 \mathrm{ml} \mathrm{m^{-1 }}$. (a) Agarose gel electrophoresis results of fragmented salmon DNA and different $\Delta P$. (b) Fragment length distributions with $A F L \approx 6 \mathrm{kbp}$ and $3 \mathrm{kbp}$ standard deviation. (c) Estimated fragmentation yields from device $D\left(N=9, w_{\mathrm{s}}=1.3 \mathrm{~mm}, h_{\mathrm{s}}=0.5 \mathrm{~mm}, w_{\mathrm{c}}=0.3 \mathrm{~mm}\right.$, $h_{\mathrm{c}}=0.2 \mathrm{~mm}, L_{\mathrm{c}}=0.5 \mathrm{~mm}, L_{\mathrm{s}}=8.5 \mathrm{~mm}$ ). 
Table 2 DNA fragmentation results. Sample concentration: $40 \mathrm{ng} \mathrm{\mu l}^{-1}$ and volume: $100 \mu \mathrm{l}$

\begin{tabular}{llllll}
\hline$Q\left(\mathrm{ml} \mathrm{min}{ }^{-1}\right)$ & AFL $(\mathrm{kbp})$ & $\gamma$ & $\mathrm{d} \varepsilon / \mathrm{d} t\left(\times 10^{5} \mathrm{~s}^{-1}\right)$ & ${ }^{c} R e$ & ${ }^{d}$ Yield (\%) \\
\hline${ }_{5}$ & 8.7 & 13.5 & 0.5 & 550 & 9 \\
${ }^{a} 10$ & 7.5 & 13.5 & 0.9 & 1110 & 40 \\
${ }_{1}{ }_{15}$ & 6.9 & 13.5 & 1.3 & 1660 & 40 \\
${ }^{a} 20$ & 6.7 & 13.5 & 1.8 & 2220 & 60 \\
${ }^{b} 40$ & 6.8 & 11 & 0.6 & 2660 & 85 \\
${ }^{b} 60$ & 6.8 & 11 & 0.9 & 3990 & 90
\end{tabular}

${ }^{a}$ Design A. ${ }^{b}$ Design D. ${ }^{c}$ Reynolds number for constriction channel using density $\rho=1.0 \mathrm{~kg} \mathrm{~s} \mathrm{~m}{ }^{-2}$ and viscosity $\eta=1.0 \times 10^{-3} \mathrm{~kg} \mathrm{~s}^{-1}$ $\mathrm{m}^{-1}$. ${ }^{d}$ The fragmentation yield is defined as the ratio of DNA fragment concentration to the non-fragmented DNA concentration.

Table 2 show DNA fragmentation results for different volumetric flow rates $Q$ and corresponding measured pressure drop $\Delta P$ in deionized water. Fig. 5 shows an example of an electrophoresis result for different designs and $\Delta P$ for $Q=60 \mathrm{ml} \mathrm{min}^{-1}$. Table 2 summarizes the experimental results.

The AFL, with $6 \mathrm{kbp}$ mean and $3 \mathrm{kbp}$ standard deviation, are similar to previously reported data using similar cross-sectional area ratio and $\Delta P .^{2}$ The lognormal AFL distribution has been previously reported, ${ }^{2,16}$ however, the exact origin of this effect is not currently known. The effect of flow on the AFL and especially the yield is evident in Table 2. The yield was further increased for a reduced $\gamma, \mathrm{d} \varepsilon / \mathrm{d} t$, and increased $Q$, which shows the strong dependence on flow rate. In our description of the fragmentation process, the flow regime was not considered, i.e. laminar, transition, or turbulent, in the constriction channels, which is $R e \sim 4000^{18}$ for $Q \sim 60 \mathrm{ml} \mathrm{m^{-1 }}$, and hence, must be at the onset of turbulence. Furthermore, the flow regime is also affected by the presence of the constriction. For many diagnostic applications larger fragment lengths are acceptable and this simple high-yield approach can be directly used. For next generation sequencing applications where sub-kbp fragment lengths are required, as previously reported, ${ }^{4}$ further device optimizations are required. Therefore, future studies will investigate the role of different flow regimes, such as turbulence, which is known to improve the fragmentation performance, ${ }^{19}$ as well as evaluating similar fragmentation geometries that can withstand higher $\Delta P$ with larger flow rates that can achieve sub-kbp average fragment lengths with high yield.

\section{Conclusions}

We have presented high yield genomic DNA fragmentation based on a new hydrodynamic shearing technique implemented in a microfluidic lab on a chip (LOC) that uses a chain of series connected constriction channels that are separated by long spacer channels with channel cross-section ratio $\gamma>10$. At each constriction channel the sample fluid accelerates and provides a shearing force for fragmentation. A sufficiently large spacing between adjacent constriction channels results in a deceleration of the sample fluid flow velocity. Repeated acceleration-deceleration cycles as the sample moves through the microfluidic structure produce a similar effect as sample recirculation, namely increasing the fragmentation yield, decreasing the fragmentation variation, and decreasing the average fragment length compared to a single constriction structure. The new method is demonstrated with a microfluidic chip that has multiple constriction channels and spacer channels and is found to improve the DNA fragmentation yield from $10 \%$ for single constriction channels up to $90 \%$ for multiple constriction channels. The new hydrodynamic shearing technique significantly simplifies the fragmentation procedure and cost, and improves the sample throughput compared to conventional hydrodynamic shearing DNA fragmentation systems.

\section{Notes and references}

1 P. N. Hengen, Trends Biochem. Sci., 1997, 22, 273.

2 L. Shui, J. G. Bomer, M. Jin, E. T. Carlen and A. van den Berg, Nanotechnology, 2011, 22, 494013.

3 J. Lichtenberg, L. Ceriotti, N. A. Lacher, S. M. Lunter, N. F. de Rooij and E. Verpoorte, Proc. $\mu$ TAS2003, California, Squaw Valley, 2003, vol. 1, p. 172.

4 I. V. Nesterova, M. L. Hupert, M. A. Witek and S. A. Soper, Lab Chip, 2012, 12, 1044.

5 Q. Tseng, A. M. Lomonosov, E. E. M. Furlonga and C. A. Merten, Lab Chip, 2012, 12, 4677.

6 M. A. Quail, DNA: Mechanical Breakage, in: Encyclopedia of Life Sciences, John Wiley \& Sons, Ltd, 2010.

7 P. J. Oefner, S. P. Hunicke-Smith, L. Chiang, F. Dietrich, J. Mulligan and R. W. Davis, Nucleic Acids Res., 1996, 24, 3879.

8 Y. R. Thorstenson, S. P. Hunicke-Smith, P. J. Oefiner and R. W. Davis, Genome Res., 1998, 8, 848.

9 B. D. Coleman, Proc. Roy: Soc. London, 1968, A306, 449.

10 G. Ryskin, J. Fluid Mech., 1987, 178, 423.

11 J. M. Wiest, L. E. Wedgewood and R. B. Bird, J. Chem. Phys., 1989, 90, 587.

12 H. R. Reese and B. H. Zimm, J. Chem. Phys., 1990, 92, 2650.

13 R. G. Larson, Rheol. Acta, 1990, 29, 371.

14 L. F. Cavalieri and B. H. Rosenberg, J. Am. Chem. Soc., 1959, 81, 5136.

15 J. A. Odell, A. Keller and A. J. Muller, Colloid Polym. Sci., 1992, $270,307$.

16 A. Joneja and X. H. Huang, BioTechniques, 2009, 46, 553.

17 F. M. White, Fluid Mechanics, 5th Ed., McGraw-Hill, 2002.

18 The Reynolds number is calculated with $R e=\rho D_{\mathrm{h}} U / \eta$, where $D_{\mathrm{h}}$ $=4 h_{\mathrm{c}} w_{\mathrm{c}} /\left(2 h_{\mathrm{c}}+2 w_{\mathrm{c}}\right)$ is the hydraulic diameter of a rectangular channel and $U=Q / h_{\mathrm{c}} \mathcal{W}_{\mathrm{c}}$ is the average constriction channel velocity.

19 S. A. Vanapalli, S. L. Ceccio and M. J. Solomon, Proc. Natl. Acad. Sci. U. S. A., 2006, 103, 16660. 